\title{
The Autonomous Concurrent Strategy for Large Scale CAE Computation
}

\author{
P. Uhruski, W. Toporkiewicz, R. Schaefer, and M. Grochowski \\ Computer Science Department, AGH University of Science and Technology, \\ Kraków, Poland \\ uhruski@ii.uj.edu.pl, Wojciech.Toporkiewicz@sheraton.com, \\ schaefer@agh.edu.pl, grochows@ii.uj.edu.pl
}

\begin{abstract}
The paper presents the Agent-Oriented technology for running the parallel CAE computation. Fast and effective distributed diffusion scheduling is available that minimizes computation and communication time necessary for task governing and provides transparency in resource availability. Detailed evaluation of the diffusion rule parameters was obtained in the course of analysis of computational, memory and communicational complexity of CAE tasks.
\end{abstract}

\section{Introduction}

Computer Aided Engineering (CAE) tasks belong to the most tiring and resource consuming. The efficient way to solve such problems is the parallel processing in a distributed environment (see e.g. [14]). We suggest the Agent-Oriented approach for implementing and governing the CAE computation in the computer network. Such system delivers much more transparency and portability in comparison to the one designed by using the traditional tools (e.g. PVM, MPI). High efficiency of such a system is caused also by the special kind of diffusion scheduling [5] that allows to adopt dynamically the computing architecture to the current resource distribution and to minimize the communication overhead. Agents being parts of such a system called Smart Solids Agents (SSA) are specially designed to follow the Subdomain-By-Subdomain (SBS) method used as the parallelization strategy (see e.g. [15]).

\section{CAE Concurrent Strategy Based on Non-overlapping Domain Decomposition}

Let us consider a computational mechanics problem over a solid domain described by a differential equation (e.g. linear elasticity stationary problem). Application of Finite Element Method (FEM) makes possible to find its approximate solution by solving of a set of linear equations $B x=f$. For FEM problems there is a parallel-processing oriented approach called Subdomain by Subdomain (SBS) 15 based on decomposition of $\Omega$ into a set of disjoined parts $\left\{\Omega_{j}\right\}$. SBS is usually combined with an iterative algorithm used for solution of resulting linear equation - Conjugate Gradient (CG) [16] in our case. 
Domain decomposition (DD) on purpose of SBS is governed by two criteria: (i) meeting memory allocation constrains for a resulting subtask; (ii) minimization of interface between subdomains (see [12,3]). Both of these criteria are evaluated in quantity of degrees of freedom (Dof) (see the next paragraph for Dof explanation) since on their number depend operating memory requirements and computation time of a resulting SBS task.

Generation of Computational Mesh (CM). CM in FEM consists of nodes grouped in elements [2]. Elements provide local support for base functions used to approximate problem's solution on the area of each of them. Dof are functionals connected with mesh nodes that define the local approximation of a solution over an element. Moreover each Dof corresponds with the single coordinate of the approximate solution $x$. Dof that are connected with CM nodes belonging to only one $\Omega_{j}$ are called internal Dof, while common to more than one - boundary Dof.

Generation of FEM matrices. As for matrix coefficients we utilize Galerkin's [15] formulation of FEM. Matrix of $j^{\text {th }}$ subdomain is of the form:

$$
\left(\begin{array}{cc}
B_{i i}^{(j)} & B_{i b}^{(j)} \\
B_{b i}^{(j)} & B_{b b}^{(j)}
\end{array}\right)\left(\begin{array}{c}
x_{i}^{(j)} \\
x_{b}^{(j)}
\end{array}\right)=\left(\begin{array}{c}
f_{i}^{(j)} \\
f_{b}^{(j)}
\end{array}\right)
$$

$B_{i i}^{(j)}$ is a block of coefficients coming exclusively from internal Dof and $B_{b b}^{(j)}$ from boundary ones while $B_{i b}^{(j)}, B_{b i}^{(j)}$ are 'mixed' blocks.

Formulation of Schur Complement System (SCS). It is possible [15, 16, to transform a global problem to one comprising only boundary components of solution $x_{b}: C x_{b}=f_{b}$ where $C=\sum_{j=1}^{s}\left(P_{b}^{(j)}\right)^{T} C^{(j)} P_{b}^{(j)}, C^{(j)}=B_{b b}^{(j)}-B_{b i}^{(j)}\left(B_{i i}^{(j)}\right)^{-1} B_{i b}^{(j)}$, $x_{b}=\sum_{j=1}^{s}\left(P_{b}^{(j)}\right)^{T} x_{b}^{(j)}, f_{b}=\sum_{j=1}^{s}\left(P_{b}^{(j)}\right)^{T}\left(f_{b b}^{(j)}-B_{b i}^{(j)}\left(B_{i i}^{(j)}\right)^{-1} f_{i}^{(j)}\right) P_{b}^{(j)}$ are mappings between the global Dof numbering scheme used in $\Omega$ and local schemes used in $\left\{\Omega_{j}\right\}$.

Solution of SCS. Provided that SCS is symmetric and positive defined CG method may be applied to obtain its solution. CG is based on minimization of the quadratic form $q(x)=\frac{1}{2} x^{T} C x-x^{T} f_{b}$ which has the unique minimizer $x$ being also the solution of SCS. CG is an iterative method where starting from the initial point $x_{0}$ at each step approximate solution is improved in the direction $d_{k}: x_{k+1}=x_{k}-\alpha_{k} d_{k}, k=0,1, \ldots$ Value for $\alpha_{k}$ can be determined by the explicit minimization of $q$ along $x_{k}-\alpha_{k} d_{k}$ so that $q\left(x_{k}-\alpha_{k} d_{k}\right)=\min _{\alpha}\left(x_{k}-\alpha d_{k}\right)$. Iteration is terminated when the distance between $x_{k}$ and $x_{k+1}$ falls below required threshold (see e.g. [16]).

Finding "internal" variables. Having computed boundary variables $x_{b}$ these referring to internal Dof can be evaluated using the formula $x_{i}^{(j)}=\left(B_{i j}^{(j)}\right)^{-1}\left(f_{i}^{(j)}\right.$ $\left.B_{i b}^{(j)} x_{b}^{(j)}\right)$. 
Domain decomposition is implemented as a recursive bisection algorithm which consists in dividing problem's domain with planes into two parts in each step and greedy optimization of interface (see [3 for details). Domain decomposition is performed sequentially on one machine. Partitioning respects both criteria (i), (ii) and the density of Dof function $\rho_{D o f}$ that provides the rough number of Dof falling into particular area $\Omega_{j}$. This part delivers minimum computational complexity $O(\log s)$ with respect to the number of subdomains $s$. This task is performed sequentially and has negligible memory complexity.

We utilize tetrahedral Delaunay 2] CM generated in parallel over $\left\{\Omega_{j}\right\}$. Computational complexity of a task in this step is between $O\left(N^{2}\right)$ and $O\left(N^{3}\right)$ where $\mathrm{N}$ is the number of Dof in particular $\Omega_{j}$. There is little communication between tasks - it is only necessary to interchange information on interfacing CM in order to assure its identity.

Computational complexity of FEM matrices generation may vary considerably depending on physical properties of a problem. There is no communication between tasks.

The key advantage of SBS consist in retaining the matrix $C$ in a distributed form of its local components $C^{(j)}$. Finding each of $C^{(j)}$ may be accomplished by inverting local matrices $B_{i i}^{(j)}$ thus computational complexity of a task in this step is not greater then $O\left(N_{i j}^{3}\right)$ where $N_{i j}$ is the number of internal Dof in particular $\Omega_{j}$. There is no communication between tasks.

The main activity of a task during CG iteration consist in multiplication of a local component of SCS by subsequent approximation of the solution vector. Total computational complexity associated with the $j^{t h}$ subdomain is $O\left(\sqrt{n} N_{b j}^{2}\right)$ in this step where $N_{b j}$ is the number of boundary Dof in $\Omega_{j} \cdot \sqrt{n}$ represents a rough evaluation of PCG iteration number while $n$ stands for the $C$ dimension (see [1]). Total communication complexity is also $O\left(\sqrt{n} N_{b j}^{2}\right)$. This part of SBS process is strongly synchronized since after each multiplication step it is necessary to assembly entire vector $x_{k}$ on the master node in order to verify coherence condition and compute direction vector $d_{k}$.

Finding internal variables can be completed in the time $O\left(N_{i j}\right)$ and communication is $O\left(N_{b j}\right)$ since only $x_{b}^{(j)}$ needs to be sent to the task associated with $\Omega_{j}$. The maximum memory complexity of above steps is $O\left(N_{i j}^{2}+N_{b j}^{2}\right)$.

Our implementation of SBS-CG process differs from the most standard approach in decomposing model of $\Omega$ on the base of $\rho_{D o f}$ rather than partitioning ready CM. This provides for straightforward parallelization of later CM generation and since computational and memory complexity of this process is square with respect to the number of nodes this strategies provide considerable savings in terms of operating memory and CPU time.

\section{The Course of the Agent-Oriented Approach}

The agent paradigm may be applied to the CAE computation to unleash any possible relaxations in the course of computation. Each task associated with $\Omega_{j}$ part of a solid is being wrapped by an autonomous agent that tries to compute 
as much independently as possible synchronizing with other tasks only when required. Agents are responsible for allocation of appropriate computational resources and running internal task work as long as no synchronization is required. Let us outline possible relaxation of consecutive steps of CAE technology:

- Mesh computation may be done autonomously with respect to the neighboring agents that need to exchange the sibling interface. That means this step requires only partial synchronization between neighboring solid parts and no global synchronization is imposed.

- Interior meshes are generated asynchronously.

- Linear equation matrix creation may be done autonomously while its assembling requires communication.

The proposed Agent-Oriented solution is based on the Octopus platform [4, which is composed of software servers statically allocated on computer nodes (hence named Virtual Computation Node - VCN) that perform information, migration and hibernation policies for mobile computing units - agents (see [7]). Every VCN maintains a basic set of operations supporting agents communication needs. It builds up the topology of neighboring VCNs that let agents examine load of sibling nodes and migrate if required using the diffusion based scheduling principle.

Such layered architecture makes the underlying network environment transparent to the agent based application. Octopus supports agent activities and provides required, unified information while hiding possibly heterogeneous environment including amount of machines, their load and network segments bandwidth. That allows agents to be executed in a heterogeneous environment. On the other hand, the computing application is composed of mobile agents that wrap computational tasks.

Octopus platform was implemented in JAVA 9] and on purpose of inter-agent communication utilizes CORBA [11] architecture.

\section{Agent-Oriented CAE Computation in the Octopus Environment}

The Smart Solid Agent (SSA) architecture [4, 8] was chosen to perform CAE type computational tasks. Its overall intention is to facilitate the design and implementation of computing application for heterogeneous and dynamic computer network.

Computing application composed of mobile SSA agents is responsible for execution of carrying task and for finishing its computation in the shortest possible time. Each Smart Solid Agent is represented by a pair $A=(T, S)$ where $T$ is the carried task including all data required for computation and $S$ is a shell responsible for the agent specific logic. The shell $S$ maintains the computational task including communication capabilities and scheduling mechanism realized by partitioning of the agent and by migration among available computers in the network. 
At the beginning the problems' domain $\Omega$ is decomposed into subdomains $\Omega_{j}$ to achieve optimal grain respecting the current computational environment. Then for every subdomain a computational task is created. In order to allow SSA to carry more then one task a special task called "task container" is utilized to wrap execution of many tasks. The agent partitioning is implemented by division of contained set of tasks and creation of two child agents with cloned shells and with new "task containers" including divided subsets of tasks.

\section{Governing of the CAE Agents}

The CAE multi-agent application starts from one agent containing all tasks coming from decomposition of the whole domain $\Omega$ of a CAE problem. The first agent as well as all its child agents search for needed resources by using the diffusion scheduling (see e.g. [5]). Roughly saying diffusion scheduling allows SSA to migrate to the least loaded VCN in its neighborhood or to be partitioned in case of insufficient resources found on the current VCN.

If an agent contains only one task it executes the task in it's own thread. For a "task container" with more then one task their execution can be performed in parallel on UMA machines.

Diffusion rules implemented in agent logic $S$ are based on the parameters $E, M, C$ collected for all tasks working in the neighborhood of each particular VCN. Meaning of such parameters is as follows: $E$ is the remaining computation time measured in units common for all computational tasks; $M$ is required RAM and $C$ characterizes communication needed for a task. Communication description $C$ is a set of pairs $(T$, data) where $T$ is an identifier of a destination task and data is amount of data exchanged betwen $T$ and the task. The momentary values of $E, M, C$ may be evaluated trough analysis of memory, time, and communication complexity of utilized algorithms.

Each CAE task (see section 2) is responsible for generation of computational mesh over associated subdomains $\Omega_{j}$, creation of FEM matrices and solving linear equations as a slave of a SBS-CG solver. Assuming featured complexities a task provides the following values required for performing diffusion scheduling, during each step of computation:

Mesh generation: $M \sim M_{1}=\beta\left(N_{i j}+N_{b j}\right)$ where $\beta$ coefficient is dependent on the shape of a subdomain; $E \sim\left(N_{i j}+N_{b j}-N_{t j}\right)^{3}$ where $N_{t j}$ is the number of vertices already included in the final mesh. Mesh generator first creates all vertices and then takes one by one and generates final mesh (when the mesh is generated $N_{t j}=N_{i j}+N_{b j}$ ); $C$ is insignificant and can be omitted. Communication is only required at the beginning of mesh generation to synchronize two-dimensional meshes on interfaces. The exact number of Dof is available when all mesh vertices have been generated. Before and during vertices generation step the number of Dof is evaluated as $\left(N_{i j}+N_{b j}\right) \simeq \int_{\Omega_{j}} \rho_{D o f}$. 
Matrix generation: $M \sim M_{2}=M_{1}+\left(N_{j j}^{2}+N_{b j}^{2}\right) ; E \sim \alpha\left(N_{i j}+N_{b j}\right)^{2}$ where $\alpha$ may be very large and may vary considerably depending on physical properties of a problem; There is no communication between tasks $(C=\phi)$.

SCS formulation: $M \sim M_{2} ; E \sim\left(N_{i j}\right)^{3} ; C=\phi$.

$C G$ iterations: $M \sim M_{2} ; E \sim \sqrt{n} N_{b j}^{2}\left(1-(1-q)^{2} t q^{t-2}\right), 0<q<1$ where $t$ is the CG iteration number; $C \sim\left\{\left(T_{m}, \sqrt{n} N_{b j}^{2}\right)\right\}$ and $T_{m}$ stands for the master node of a SBS-CG solver.

Finding internal variables: $M \sim M_{2} ; E \sim N_{i j} ; C \sim\left\{\left(T_{m}, N_{b j}\right)\right\}, j=1, \ldots, s$.

\section{Numerical Test}

Initially performed tests intend to show if the diffusion based strategy for the first two phases of CAE properly utilized available computational resources and provided satisfactory speedup. Our computing environment was composed of up to $10 \mathrm{PC}$ machines with Intel Celeron processors and 512MB RAM. Every machine hosted one VCN application (an Octopus computation node). As the underlying operating system we selected the SLAX Linux operating system [10]. This Linux distribution was selected basing on the SLAX ability to boot up from a bootable CD. That way we were able to link an available IBM PC compliant machine into the computational platform without additional installation. Each booted machine completed a star-like Octopus virtual topology by connecting to one selected root machine. Hence we obtained a cluster-like environment of 10 machines all connected by a fast LAN network.

An example solid was selected (see figure1) and pre-partitioned. That resulted in 14 tasks (12 similar in size and 2 slightly larger ones), each related to a

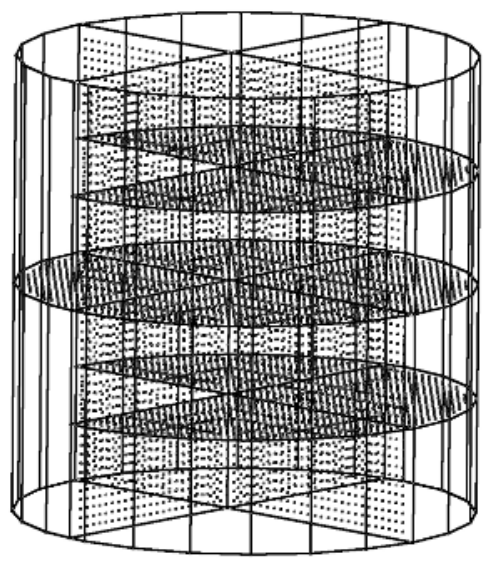

Fig. 1. Decomposed domain of the problem with visible interface meshes 
Table 1. Speed up of mesh generation for different values of Dof function $\rho_{D o f}$ representing mesh density

\begin{tabular}{|c|c|c|c|c|c|}
\hline \multirow{2}{*}{$\begin{array}{c}\text { Mesh } \\
\text { density }\end{array}$} & Number of & \multirow{2}{*}{$\begin{array}{c}\text { Number of } \\
\text { vertices }\end{array}$} & \multicolumn{2}{|c|}{$\begin{array}{c}\text { Execution time [sec] } \\
\text { elements }\end{array}$} & Speed up \\
\hline \hline 0,50 & 93420 & 535380 & 815 & 134 & 6,08 \\
\hline 0,75 & 313523 & 1823385 & 5492 & 806 & 6,81 \\
\hline 0,85 & 377562 & 2721561 & 10986 & 1595 & 6,89 \\
\hline 1,10 & 975482 & 5823631 & 40613 & 5856 & 6,94 \\
\hline
\end{tabular}

single piece of the solid. In the course of computations all machines were used by the agents thus $100 \%$ resources were allocated. The table 1 presents the speedup gained by the mesh generation agents versus serial computation time. Explanation of the achieved speedup comes from the number of tasks and the number of utilized machines. During the computation 6 machines were executing one task each (the larger tasks were executed by these), while other 4 machines were running two, similar in size, tasks each. We had 8, similar in size tasks executed in pairs by four machines. That means the maximum achieved speedup could be half of the tasks amount. Finally please note that the overall parallel time results from the the slowest tasks and since all tasks were similar in size, the overall speedup could be half of the tasks amount and that is 7 .

\section{Conclusions}

The paper presents Agent-Oriented technology for running parallel CAE computations in a computer network. The approach provides fast and effective distributed diffusion scheduling that minimizes computation and communication time necessary for task governing. Moreover resources in a dynamic network environment are transparently available. (see [8, 5, 7] for more test results). Detailed evaluation of the diffusion rule parameters were obtained on the basis of computational, memory and communicational complexity analysis of CAE tasks. The numerical test presented in the section 7 shows behavior of agents performing two initial phases of CAE process - domain decomposition and mesh generation.

\section{References}

1. Barragy E., Carey G.F., Van de Geijn R.: Performance and Scalability of Finite Element Analysis for Distributed Parallel Computation. Journal of Parallel and Distributed Computing 21, (1994) pp. 202-212.

2. Georg P.L.: Automatic Mesh Generation. John Wiley \& Sons, 1991. 
3. Schaefer R., Toporkiewicz W., Grochowski M.: Rough partitioning of lumped structures, in Formal Methods and Intelligent Techniques in Control, Decision Making, Multimedia and Robotics. Polish-Japanese Institute of Information Technology Press, Warsaw, October 2000, pp. 151-166.

4. Grochowski M., Schaefer R., Uhruski P.: An Agent-based Approach To a Hard Computing System - Smart Solid. Proc. of the International Conference on Parallel Computing in Electrical Engineering (PARELEC 2002), 22-25 September 2002, Warsaw, Poland. IEEE Computer Society Press 2002, pp. 253-258.

5. Grochowski M., Schaefer R., Uhruski P.: Diffusion Based Scheduling in the AgentOriented Computing Systems. Lecture Notes in Computer Science, Vol. 3019, Springer 2004, pp. 97-104.

6. Momot J., Kosacki K., Grochowski M., Uhruski P., Schaefer R.; Multi-Agent System for Irregular Parallel Genetic Computations. Lecture Notes in Computer Science, Vol. 3038, Springer 2004, pp. 623-630.

7. Smoka M., Uhruski P., Schaefer R., Grochowski M.; The Dynamics of Computing Agent Systems. Lecture Notes in Computer Science Vol. 3516, Springer 2005, pp. 727-734.

8. Uhruski P., Grochowski M., Schaefer R.: Multi-agent Computing System in a Heterogeneous Network. Proc. of the International Conference on Parallel Computing in Electrical Engineering (PARELEC 2002), 22-25 September 2002, Warsaw, Poland. IEEE Computer Society Press 2002, pp. 233-238.

9. Sun Microsystems, Java Technology, http://java.sun.com/

10. SLAX, SLAX Linux operating system, http://slax.linux-live.org/

11. CORBA, Object Management Group, http://www.omg.org/

12. Schaefer R., Flasiński M., Toporkiewicz W.: Optimal Stochastic Scaling of CAE Parallel Computations. Lecture Notes in Computer Intelligence, Vol. 1424, Springer 1998, pp.557-564.

13. Norton C. D., Cwik T. A.: Parallel Unstructured AMR and Gigabit Networking for Beowulf-Class Clusters. Lecture Notes in Computer Science, Vol. 2328, SpringerVerlag Heidelberg 2002, pp. 552-563.

14. Mann V., Parashar M.: Engineering an interoperable computational collaboratory on the Grid. Concurrency and Computation. Practice and Experience., 14, pp. 1569-1593, 2002.

15. Papadrakakis M.: Domain decomposition techniques in Computational Structural Mechanics in M. Papadrakakis (Ed.), Parallel Solution Methods in Computational Mechanics, John Wiley and Sons (1996), pp. 87-140.

16. Golub G., Ortega J.M.: Scientific Computing. An Introduction with Parallel Computing, Academic Press Ltd., 1993. 